\title{
Measurement of the hadronic cross sections at Novosibirsk
}

\author{
I.B.Logashenko ${ }^{1,2, a}$, M.N.Achasov ${ }^{1,2}$, R.R.Akhmetshin ${ }^{1}$, A.N.Amirkhanov², A.V.Anisenkov ${ }^{1,2}$, V.M.Aulchenko ${ }^{1,2}$, \\ V.S.Banzarov ${ }^{1}$, N.S.Bashtovoy ${ }^{1}$, A.Yu. Barnyakov1, K.I. Beloborodov1, A.V. Berdyugin ${ }^{1}$, D.E.Berkaev ${ }^{1,2}$, A.G.

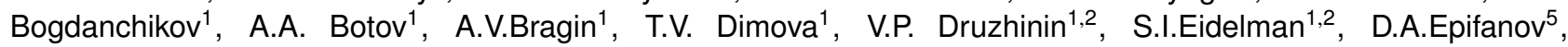 \\ L.B.Epshteyn ${ }^{1,3}$, A.L.Erofeev ${ }^{1}$, G.V.Fedotovich ${ }^{1,2}$, S.E.Gayazov ${ }^{1,2}$, V.B. Golubev ${ }^{1,2}$, A.A.Grebenuk ${ }^{1,2}$, \\ D.N.Grigoriev ${ }^{1,2,3}$, E.M.Gromov ${ }^{1}$, F.V.Ignatov ${ }^{1}$, L.V. Kardapoltsev ${ }^{1,2}$, S.V.Karpov ${ }^{1}$, V.F.Kazanin ${ }^{1,2}$, A.G. Kharlamov $^{1,2}$, \\ I.A.Koop ${ }^{1,2}$, A.A. Korol ${ }^{1,2}$, S.V. Koshuba ${ }^{1}$, O.A.Kovalenko ${ }^{1,2}$, D.P. Kovrizhin ${ }^{1,2}$, A.N.Kozyrev ${ }^{1}$, E.A.Kozyrev ${ }^{1,2}$, \\ P.P.Krokovny, ${ }^{1,2}$, A.S. Kupich ${ }^{1}$, A.E.Kuzmenko ${ }^{1,3}$, A.S.Kuzmin ${ }^{1}$, P.A.Lukin ${ }^{1,2}$, K.A. Martin ${ }^{1,2}$, K.Yu.Mikhailov ${ }^{1,2}$, A.E. \\ Obrazovsky $^{1}$, V.S.Okhapkin1, A.V. Otboev1, E.V. Pakhtusova1, Yu.N.Pestov1, A.S.Popov ${ }^{1,2}$, G.P.Razuvaev ${ }^{1,2}$, \\ Yu.A.Rogovsky1, A.L.Romanov ${ }^{1}$, A.A.Ruban ${ }^{1}$, N.M.Ryskulov ${ }^{1}$, A.E.Ryzhenenkov ${ }^{1,2}$, S.I. Serednyakov ${ }^{1,2}$, \\ V.E.Shebalin ${ }^{1}$, D.N.Shemyakin ${ }^{1,2}$, D.A. Shtol ${ }^{1}$, B.A.Shwartz ${ }^{1,2}$, D.B.Shwartz ${ }^{1,2}$, A.L.Sibidanov ${ }^{4}$, Z.K. Silagadze ${ }^{1,2}$, \\ P.Yu.Shatunov ${ }^{1}$, Yu.M.Shatunov ${ }^{1}$, E.P.Solodov ${ }^{1,2}$, I.K. Surin ${ }^{1}$, V.M.Titov ${ }^{1}$, A.A.Talyshev ${ }^{1,2}$, A.V. Vasiljev $^{1}$, \\ A.I.Vorobiov ${ }^{1}$, and Yu.V.Yudin ${ }^{1}$ \\ ${ }^{1}$ Budker Institute of Nuclear Physics, SB RAS, Novosibirsk, 630090, Russia \\ ${ }^{2}$ Novosibirsk State University, Novosibirsk, 630090, Russia \\ ${ }^{3}$ Novosibirsk State Technical University, Novosibirsk, 630092, Russia \\ ${ }^{4}$ University of Sydney, School of Physics, Falkiner High Energy Physics, NSW 2006, Sydney, Australia \\ ${ }^{5}$ University of Tokyo, Department of Physics, 7-3-1 Hongo Bunkyo-ku Tokyo, 113-0033, Japan
}

\begin{abstract}
Two experiments, CMD-3 and SND, collected over $100 \mathrm{pb}^{-1}$ in total taking data in 2011-2013 at the VEPP-2000 electron-positron collider. The data sample covers the whole available c.m. energy range from 0.32 $\mathrm{GeV}$ to $2.0 \mathrm{GeV}$. An upgrade of VEPP-2000 started in the second half of 2013, aimed at the luminosity increase up to 10 times at $2.0 \mathrm{GeV}$. Data taking is expected to resumed in 2015 . The current results of analysis of the collected data for various modes of $e^{+} e^{-} \rightarrow$ hadrons are discussed.
\end{abstract}

\section{VEPP-2000}

The electron-positron collider VEPP-2000[1, 2] was commissioned at Budker Institute of Nuclear Physics (Novosibirsk, Russia) in 2010. The machine covers the c.m. energy range from $\sqrt{s}=0.32 \mathrm{GeV}$ to $2.0 \mathrm{GeV}$ and employs the novel technique of round beams to reach a design luminosity up to $10^{32} \mathrm{~cm}^{-2} \mathrm{~s}^{-1}$ at $2 \mathrm{GeV}$.

Two detectors, CMD-3[3] and SND[4], are installed in the interaction regions of VEPP-2000.

CMD-3 (Cryogenic Magnetic Detector) is a generalpurpose detector (Fig. 1). The cylindrical drift chamber with hexagonal cells is surrounded by the Z-chamber, a MWPC with a dual anode and cathode readout, used for precise determination of the fiducial volume for charged particles. The barrel electromagnetic calorimeter, placed outside the superconducting solenoid $\left(0.13 \mathrm{X}_{0}, 13 \mathrm{kGs}\right)$, is composed of two systems: the Liquid Xenon calorimeter (about $5.4 \mathrm{X}_{0}$ ), surrounded by the CsI crystal calorimeter (about $8.1 \mathrm{X}_{0}$ ). The LXe calorimeter has 7 layers and utilizes dual readout: the anode signals are used for a measurement of the total energy deposition, while signals from the cathode strips provide information about a shower pro-

\footnotetext{
ae-mail: I.B.Logashenko@inp.nsk.su
}

file and are used for a measurement of photon coordinates with high precision (about 1-2 mm). The endcap BGO crystal calorimeter (about $13.4 \mathrm{X}_{0}$ ) operates in the main magnetic field. The time-of-flight system, designed to identify slow moving particles, is placed between the two layers of the barrel calorimeter. The detector is surrounded by the muon range system.

The SND (Fig.2) is a general-purpose nonmagnetic detector. It consists of the 9-layer drift chamber, the aerogel Cherenkov counters, the three-layer spherical electromagnetic calorimeter with $1680 \mathrm{NaI}(\mathrm{Tl})$ crystals and the muon system.

The physics program of experiments includes the high precision measurement of cross sections of various modes of $e^{+} e^{-} \rightarrow$ hadrons, studies of known and searches for new vector mesons, measurement of $n \bar{n}$ and $p \bar{p}$ production cross sections near threshold and searches for exotic hadrons.

Measurement of the total cross section of $e^{+} e^{-} \rightarrow$ hadrons in the VEPP-2000 energy range is very interesting in relation with the problem of anomalous magnetic moment of muon, $a_{\mu}$.

The most recent measurement of $a_{\mu}$ done at BNL with $0.54 \mathrm{ppm}$ precision [5] gave a very interesting outcome: 


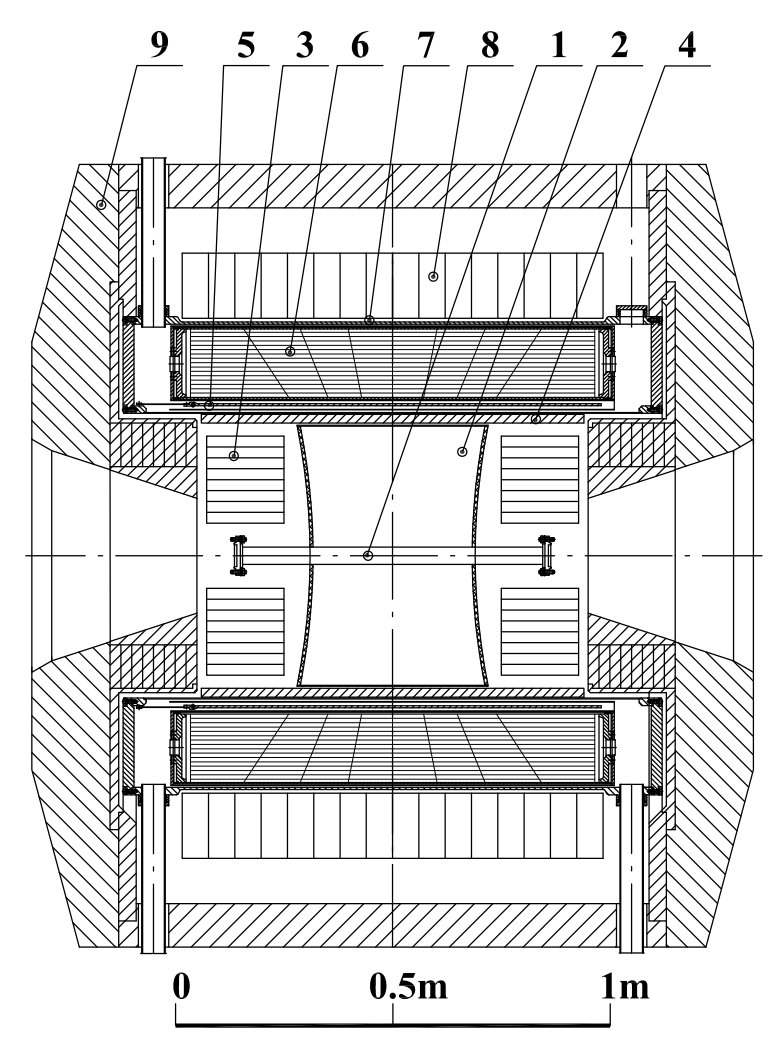

Figure 1. CMD-3 detector: 1 - beam pipe, 2 - drift chamber, 3 BGO calorimeter, 4 - Z-chamber, 5 - superconducting solenoid, 6 - LXe calorimeter, 7 - TOF system, 8 - CsI calorimeter, 9 yoke. The muon range system, not shown, is placed outside the yoke.

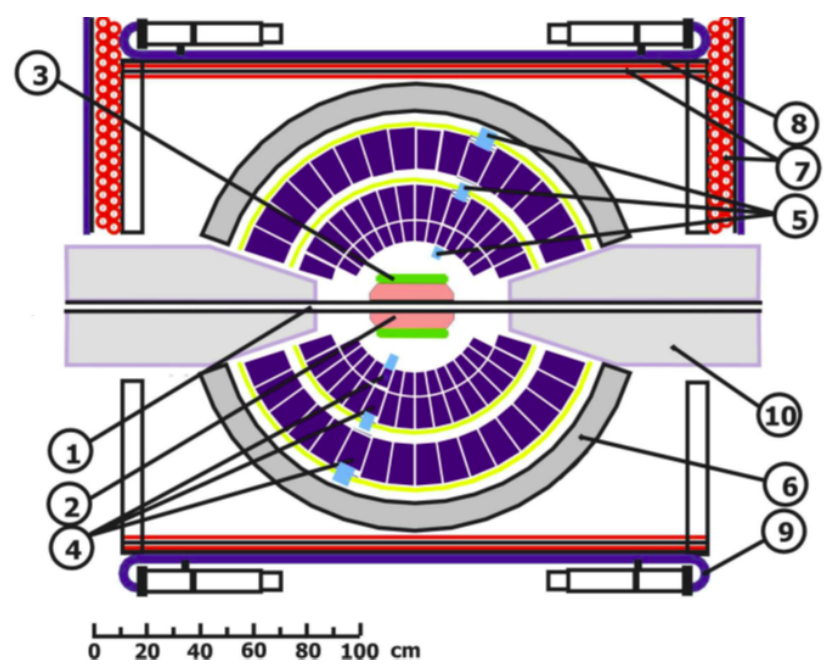

Figure 2. The SND detector: 1 - beam pipe, 2 - tracking system, 3 - aerogel Cherenkov counters, 4 - NaI(Tl) crystals, 5 - phototriodes, 6 - iron muon absorber, 7-9 - muon detector, 10 - focusing superconducting solenoids. the measured value is $2.2 \div 2.5 \mathrm{ppm}$, or $3.3 \div 3.6$ standard deviations above the Standard Model expectation. All existing fields contribute to $a_{\mu}$ via loop corrections. While the QED and EW contributions to $a_{\mu}$ can be calculated using perturbative approach with necessary precision, the hadronic contribution cannot, due to non-perturbative nature of QCD. The most precise calculation of the hadronic contribution $a_{\mu}(\mathrm{had})$ is obtained with the technique, based on the dispersion relation, where the lowest-order value $a_{\mu}(h a d ; L O)$ is obtained by integration of $R(s)$, the ratio of $\sigma\left(e^{+} e^{-} \rightarrow\right.$ hadrons $)$ to $\sigma\left(e^{+} e^{-} \rightarrow \mu^{+} \mu^{-}\right)$, with a proper kernel:

$$
a_{\mu}^{h a d ; L O}=\left(\frac{\alpha m_{\mu}}{3 \pi}\right)^{2} \int_{m_{p} i^{2}}^{\infty} \frac{d s}{s^{2}} K(s) R(s) .
$$

Due to the $1 / s^{2}$ factor, the major part of integral comes from low energies. In particular, the VEPP-2000 energy range contributes $>90 \%$ to $a_{\mu}(h a d ; L O)$, with a dominant (>70\%) contribution from the $e^{+} e^{-} \rightarrow \pi^{+} \pi^{-}$final state.

One of the main goals of experiments at VEPP-2000 is to measure $R(s)$ with high precision. In the VEPP-2000 energy range, due to small multiplicity, the only viable option is to measure each final state exclusively. The more a particular final state contributes to the (g-2) integral (1), the smaller systematic error is required. The most stringent requirements for accuracy are set for the $e^{+} e^{-} \rightarrow$ $\pi^{+} \pi^{-}$final state, and, to less extent, for $e^{+} e^{-} \rightarrow 4 \pi$.

\section{Data taking history}

The data were taken in three independent seasons. In 2011 and 2012 seasons the data were collected in the c.m. energy range from $1.0 \mathrm{GeV}$ to $2.0 \mathrm{GeV}$. About $30 \mathrm{pb}^{-1}$ were collected per detector, with about $10 \mathrm{pb}^{-1}$ per detector above the $p \bar{p}$ threshold. The 2013 season was dedicated to energy scan below $1.0 \mathrm{GeV}$, down to $0.32 \mathrm{GeV}$ in c.m., the lowest collision energy ever achieved. About $20 \mathrm{pb}^{-1}$ were collected per detector, with about $8 \mathrm{pb}^{-1}$ per detector near the peak of the $\omega(782)$.

The beam energy monitoring system[6] has been installed and commissioned during the 2012 season. The system allows to continuously monitor beam energy with relative precision $<10^{-4}$, using Compton backscattering of laser photons on the electron beam.

Average VEPP-2000 luminosity as a function of beam energy is shown in Fig. 3. The luminosity achieved at energies above $1.2 \mathrm{GeV}$ is notably lower than the design luminosity, up to 10 times at $\sqrt{s}=2.0 \mathrm{GeV}$. There is a number of factors contributing to this deficiency. At energies $\sqrt{s} \approx 1.2 \mathrm{GeV}$ and above, the luminosity is limited by the rate of positron production. The maximum energy of the booster ring is $0.825 \mathrm{GeV}$, thus at energies $\sqrt{s} \geq 1.65 \mathrm{GeV}$ the VEPP-2000 energy has to be ramped up and down for each injection, which further limits the effective luminosity.

In order to overcome these limitations, an upgrade of VEPP-2000 started in the second half of 2013. The upgrade plan includes a number of improvements: switching over to the new, at least ten times more productive beam 


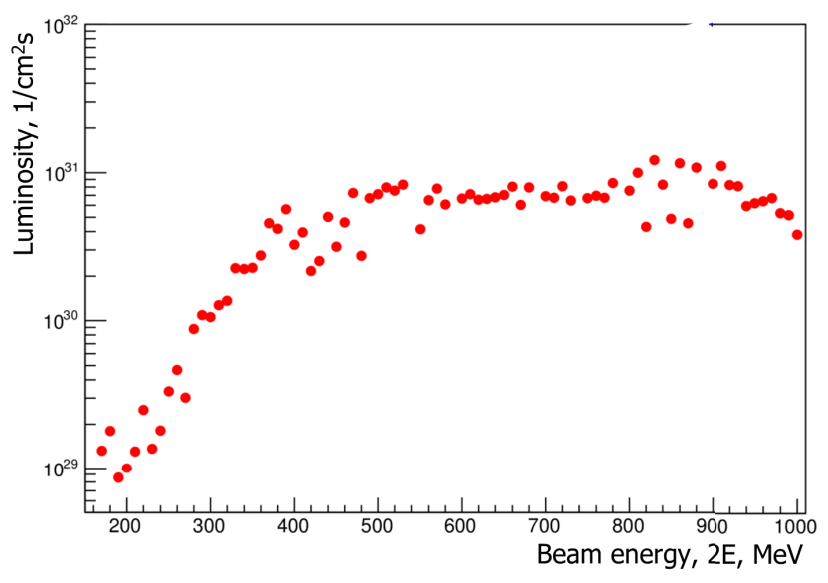

Figure 3. Average VEPP-2000 luminosity as a function of beam energy, measured by the CMD-3 detector.

source; complete rearrangement of the booster ring to increase it maximum energy to $2.0 \mathrm{GeV}$; and other smaller projects. The upgrade is still under way and data taking is expected to be resumed in the second half of 2015.

In the following section we review the recent results of analysis of data, taken with CMD-3 and SND in 20112013.

\section{Results of data analysis}

\subsection{Cross section of $e^{+} e^{-} \rightarrow \pi^{+} \pi^{-}$}

As was mentioned before, the simplest mode of $e^{+} e^{-} \rightarrow$ hadrons, the process $e^{+} e^{-} \rightarrow \pi^{+} \pi^{-}$, plays special role in the calculation of $a_{\mu}$.

The cross section $e^{+} e^{-} \rightarrow \pi^{+} \pi^{-}$was measured to high precision in a number of experiments. The most precise direct energy scan measurements were performed in the previous generation of experiments in Novosibirsk at the VEPP-2M collider with the CMD-2 and SND detectors. The high precision measurements of this mode were recently performed in KLOE and BaBar experiments using initial-state radiation (ISR) technique. The systematic error of all these measurements varies from $0.5 \%$ to $1.3 \%$ in the $\rho(770)$ resonance energy range. The reviews of the existing data are provided in the most recent calculations of the hadronic contribution to $a_{\mu}[7,8]$ and in [9].

There is good agreement of the value of the $(g-2)$ integral (1) in the discussed measurements, but when their results are compared in different energy regions, there are local discrepancies of a few percent, far beyond the quoted levels of the statistical and systematic errors.

In 2013 the energy scan of the energy range between $0.32 \mathrm{GeV}$ and $1.0 \mathrm{GeV}$ was performed, dedicated to $R(s)$ measurement. Here we discuss the preliminary results of the measurement of the $e^{+} e^{-} \rightarrow \pi^{+} \pi^{-}$cross section at CMD-3. The CMD-3 ultimate goal it to measure this cross section with systematic uncertainty of $0.35 \%$ at $\sqrt{s}<1$ $\mathrm{GeV}$ and moderately larger uncertainty at higher energies, which is about factor of 2 improvement compared with

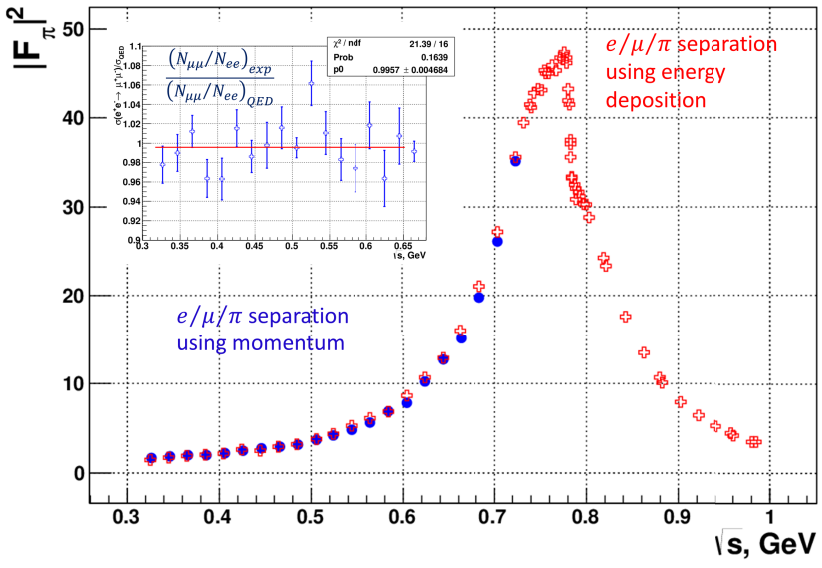

Figure 4. Preliminary results of pion formfactor measurement at CMD-3. Red crosses correspond to $e / \mu / \pi$ separation using the energy deposition in the calorimeter, blue circles correspond to $e / \mu / \pi$ separation using the momentum. The inset shows the ratio of the measured $e^{+} e^{-} \rightarrow \mu^{+} \mu^{-}$cross section to the QED expectation for the blue circles.

the previous measurements at VEPP-2M and similar to the precision of the today's world average.

The events with two back-to-back tracks are selected. This sample includes $e^{+} e^{-} \rightarrow e^{+} e^{-}, \mu^{+} \mu^{-}$and $\pi^{+} \pi^{-}$events with practically no background except for the percent level of cosmic background. The particle identification is performed using two techniques. In the first approach, the two-dimensional distribution of the momenta of two particles is approximated by the model, which takes into account the radiative corrections and the detector response function. The momentum resolution of the CMD-3 drift chamber is enough to use this approach up to energies $\sqrt{s} \approx 0.66 \mathrm{GeV}$.

In the second approach the two-dimensional distribution of the energy deposition of two particles is used. This technique is more complicated, as there is no good theoretical model for the energy deposition of pions and stopped muons in the calorimeter. The corresponding distributions are extracted from the data using tagged muons and pions from cosmic events and decays $\omega \rightarrow \pi^{+} \pi^{-} \pi^{0}$ and $\varphi \rightarrow \pi^{+} \pi^{-} \pi^{0}$. The advantage of this approach is that it can be used in the whole VEPP-2000 energy range.

A very preliminary result of $e^{+} e^{-} \rightarrow \pi^{+} \pi^{-}$cross section measurement at CMD-3 is shown in Fig. 4 in terms of the pion formfacftor, related to the cross section as

$$
\sigma\left(e^{+} e^{-} \rightarrow \pi^{+} \pi^{-}\right)=\frac{\pi \alpha^{2}}{3 s}\left(1-\frac{4 m_{\pi}^{2}}{s}\right)^{3 / 2}\left|F_{\pi}\right|^{2} .
$$

The results of both techniques are shown. There is good agreement between the results of two approaches in the energy range $\sqrt{s} \leq 0.66 \mathrm{GeV}$, where they both can be used. It should be noted that not all necessary corrections are applied yet. Also, we are working on improvement of the model for energy deposition and on incorporating the data on the shower profile, measured in the layered barrel calorimeter, to the $e / \mu / \pi$ separation algorithm. 


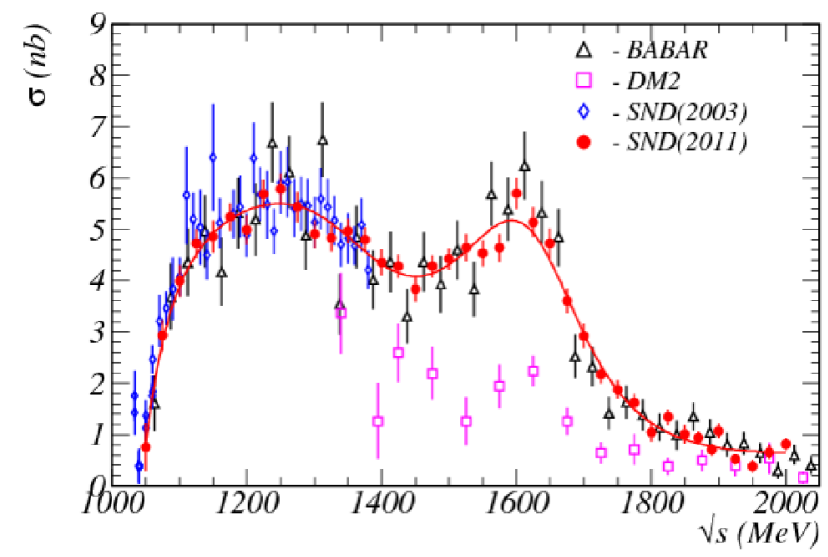

Figure 5. Cross section of the $e^{+} e^{-} \rightarrow \pi^{+} \pi^{-} \pi^{0}$ process obtained at the SND detector in comparison with previous SND and BABAR data

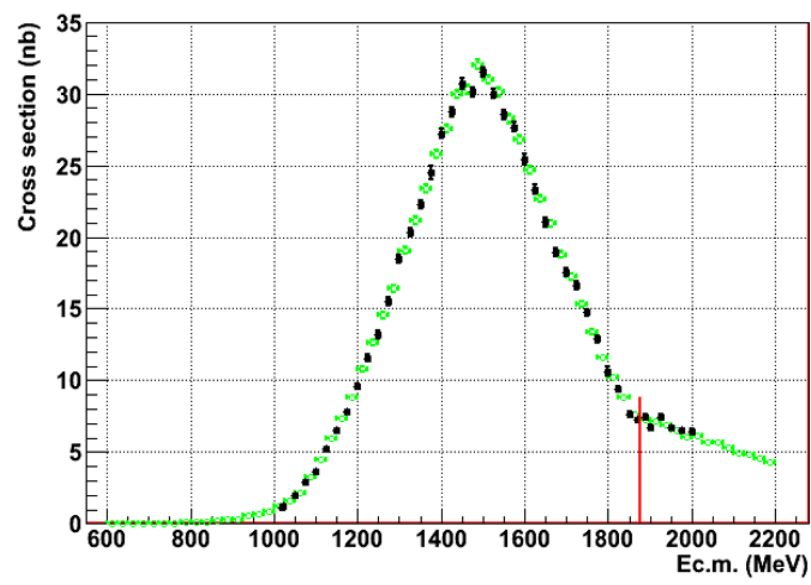

Figure 6. The preliminary result of measurement of the cross section $e^{+} e^{-} \rightarrow \pi^{+} \pi^{-} \pi^{+} \pi^{-}$at CMD-3 in comparison with BABAR data. The vertical line shows the $N \bar{N}$ threshold.

In addition to the $e^{+} e^{-} \rightarrow \pi^{+} \pi^{-}$cross section, the described procedure allows to measure the $e^{+} e^{-} \rightarrow \mu^{+} \mu^{-}$ cross section. The ratio between the measured $\sigma_{\mu \mu}$ cross section and QED expectation is shown in the inset in Fig. 4. Agreement at the $0.5 \%$ level is observed, which corresponds to the statistical accuracy. Measurement of the $e^{+} e^{-} \rightarrow \mu^{+} \mu^{-}$cross section provides a powerful test of the overall procedure.

\subsection{Cross sections $e^{+} e^{-} \rightarrow 3 \pi, 4 \pi, 5 \pi, 6 \pi$}

The cross section $e^{+} e^{-} \rightarrow 3 \pi$ measured by SND is shown in Fig. 5. It is in good agreement with results of the previous measurements by SND[10] and BABAR[11], and has better precision. The model takes into account contributions from the $\omega(782), \phi(1020), \omega^{\prime}(1450)$ and $\omega^{\prime \prime}(1680)$ resonances. The $\omega^{\prime}(1450)$ is observed in this final state only.

The preliminary result of $e^{+} e^{-} \rightarrow \pi^{+} \pi^{-} \pi^{+} \pi^{-}$cross section measurement at CMD-3 is shown in Fig. 6. The statistical error is about $1 \div 2 \%$ at each energy point. This

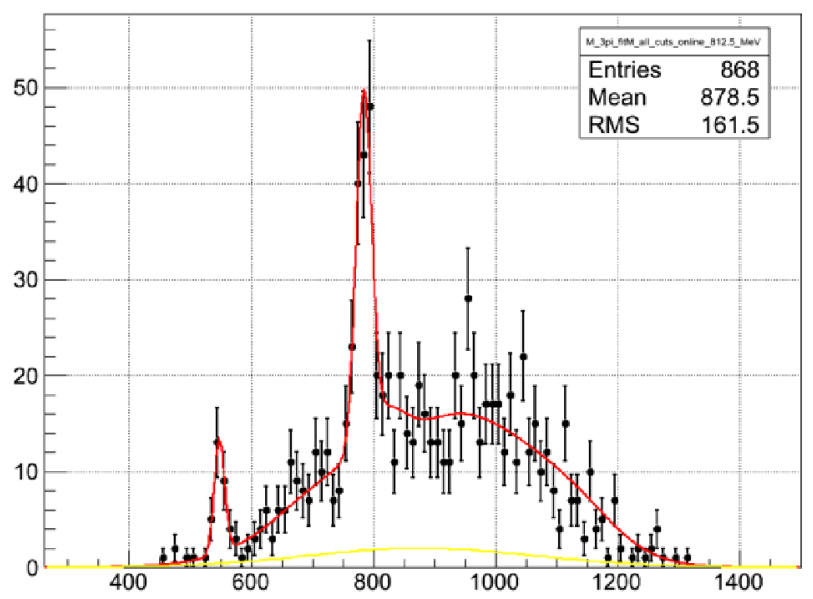

Figure 7. The distribution of invariant mass of all possible combinations of $\pi^{+} \pi^{-} \pi^{0}$ for the $\pi^{+} \pi^{-} \pi^{+} \pi^{-} \pi^{0}$ final state, data by CMD-3. There are clear signs of $\omega$ and $\eta$ with a small additional non- $\omega / \eta$ contribution.

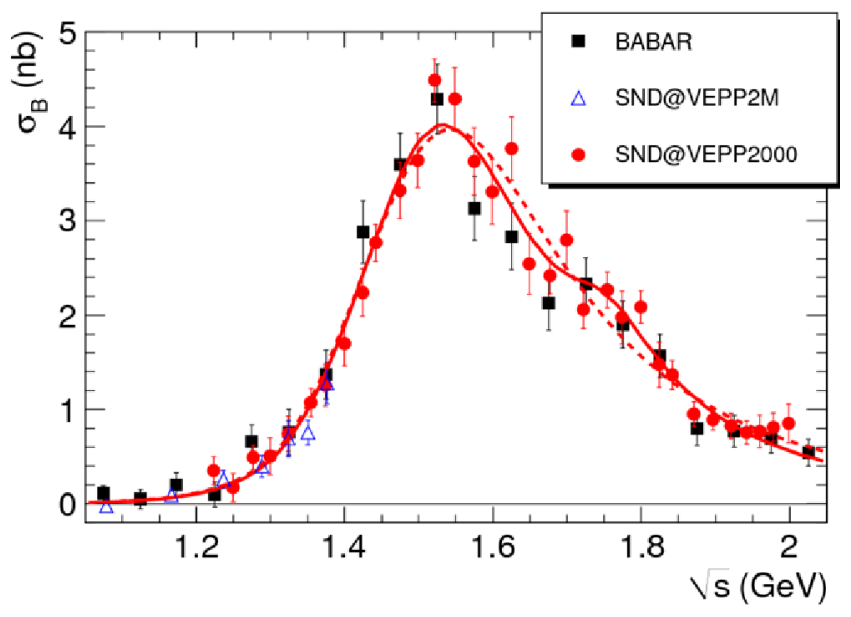

Figure 8. Cross section of $e^{+} e^{-} \rightarrow \eta \pi^{+} \pi^{-} \rightarrow \gamma \gamma \pi^{+} \pi^{-}$measured by SND. The model takes into account the $\rho(770) \eta$ and $\rho(1450) \eta$ intermediate states.

is the dominant cross section at energies above $\varphi(1020)$, therefore it is important to achieve a small systematic error of a few percent. In order to evaluate the detection efficiency (detector acceptance), the internal dynamics of this process have to be taken into account. For $e^{+} e^{-} \rightarrow$ $\pi^{+} \pi^{-} \pi^{+} \pi^{-}$, the production mechanism through $a_{1}(1260) \pi$ is confirmed, while small admixtures of $\rho(770) f_{0}(600)$, $\rho(770) f_{0}(980)$ and $a_{2}(1320) \pi$ are also seen. There is a clear signal of $\omega \pi^{0}$ events in the collected $e^{+} e^{-} \rightarrow$ $\pi^{+} \pi^{-} \pi^{0} \pi^{0}$ sample. Non- $\omega \pi^{0}$ events are dominated by $a_{1}(1260) \pi$ with additional small contributions from $\rho^{+} \rho^{-}$ and $\rho(770) f_{0}(980)$. In order to measure $\sigma\left(e^{+} e^{-} \rightarrow 4 \pi\right)$, all these production mechanisms should be taken into account in simulation.

The dominant production mechanisms for the $e^{+} e^{-} \rightarrow$ $\pi^{+} \pi^{-} \pi^{+} \pi^{-} \pi^{0}$ process are $e^{+} e^{-} \rightarrow \omega \pi^{+} \pi^{-}$and $e^{+} e^{-} \rightarrow$ $\eta \pi^{+} \pi^{-}$(Fig. 7). Both modes are clearly seen by CMD-3 


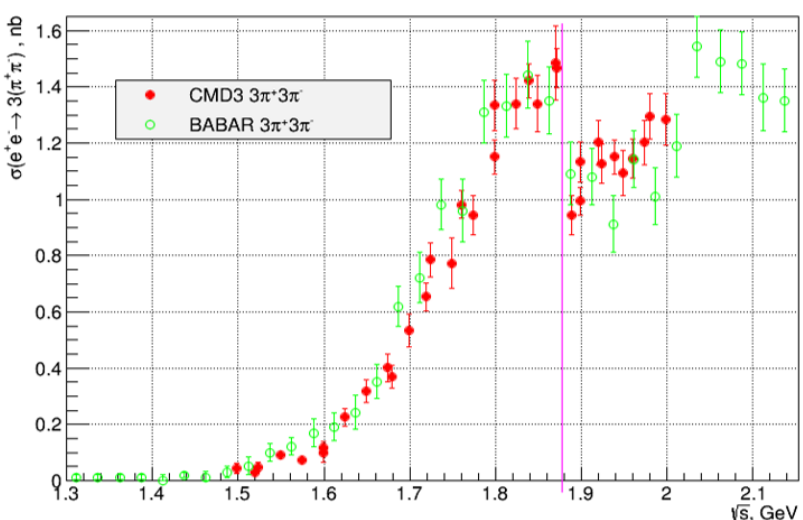

Figure 9. Cross section of the $e^{+} e^{-} \rightarrow 3\left(\pi^{+} \pi^{-}\right)$final state measured by the CMD- 3 detector. The vertical line shows the $N \bar{N}$ threshold.

and are under analysis (Fig. 7). The $e^{+} e^{-} \rightarrow \eta \pi^{+} \pi^{-}$cross section was recently measured by SND in the different final state $e^{+} e^{-} \rightarrow \eta \pi^{+} \pi^{-} \rightarrow \gamma \gamma \pi^{+} \pi^{-}$(Fig. 8).

The cross section $e^{+} e^{-} \rightarrow 3\left(\pi^{+} \pi^{-}\right)$has been recently measured at the CMD-3 detector in the $\sqrt{s}=1.5-2.0 \mathrm{GeV}$ energy range[12] (Fig. 9). It was found that the observed production mechanism can be described by the production of one $\rho(770)$ with four remaining pions in the S-wave distributed according to phase space. The interesting feature of the measured cross section is a sharp dip at the $p \bar{p}$ threshold. It was shown [13] that this dip is compensated by opening of the $p \bar{p}$ and $n \bar{n}$ modes. Detailed analysis of the production dynamics will be performed in the combined analysis of the processes $e^{+} e^{-} \rightarrow 3\left(\pi^{+} \pi^{-}\right)$and $e^{+} e^{-} \rightarrow 2\left(\pi^{+} \pi^{-} \pi^{0}\right)$.

\subsection{Final states with kaons}

At energies above the $\varphi(1020)$, final states with kaons add a significant contribution to $e^{+} e^{-} \rightarrow$ hadrons. Charged kaons are well identified at CMD- 3 by large $\mathrm{dE} / \mathrm{dX}$ in the drift chamber, and at SND using Cherenkov counters.

The preliminary results of $e^{+} e^{-} \rightarrow K^{+} K^{-}$and $e^{+} e^{-} \rightarrow$ $K_{S} K_{L}$ cross section measurement near the $\varphi(1020)$ peak at CMD-3 are shown in Fig. 10. The systematic error of this measurement is estimated to be around $2 \%$. In earlier experiments [14] it was observed that the ratio $g_{\varphi \rightarrow K^{+} K^{-}} / g_{\varphi \rightarrow K_{S} K_{L}}$ differs from the theoretical expectation by more that $3 \sigma$. The new simultaneous measurement of these cross sections at CMD-3 confirms the discrepancy. The preliminary results of measurement of the $e^{+} e^{-} \rightarrow K^{+} K^{-}$cross section at energies above the $\varphi(1020)$ peak done by SND are shown in Fig. 11.

Preliminary results of CMD-3 analysis of the $e^{+} e^{-} \rightarrow$ $K^{+} K^{-} \pi^{+} \pi^{-}$final state are shown in Fig. 12. This mode is particularly interesting because many intermediate states contribute to it: $K^{*}(1270) K \rightarrow K^{*}(892) K \pi, K^{*}(1400) K \rightarrow$ $K^{*}(892) K \pi, K^{*}(1270) K \rightarrow \rho K K, K^{*}(892) K^{*}(892), \phi \pi \pi$.

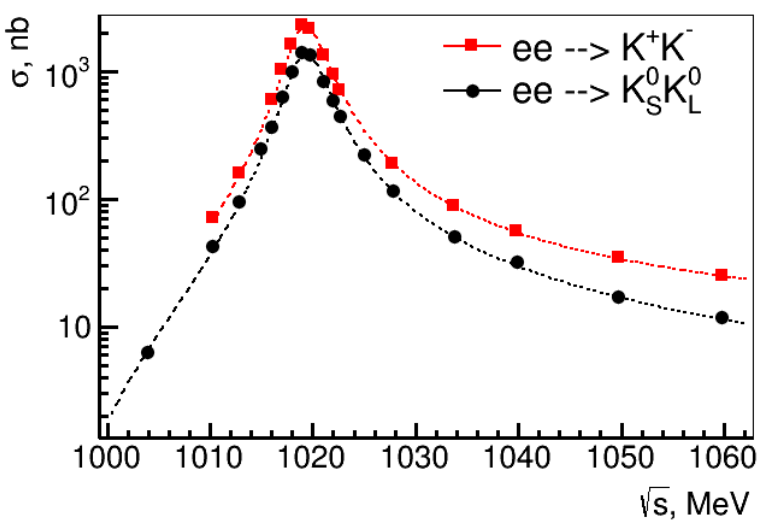

Figure 10. Preliminary results of measurement of the $e^{+} e^{-} \rightarrow$ $K^{+} K^{-}$and $e^{+} e^{-} \rightarrow K_{S} K_{L}$ cross sections near the $\varphi(1020)$ peak at CMD-3.

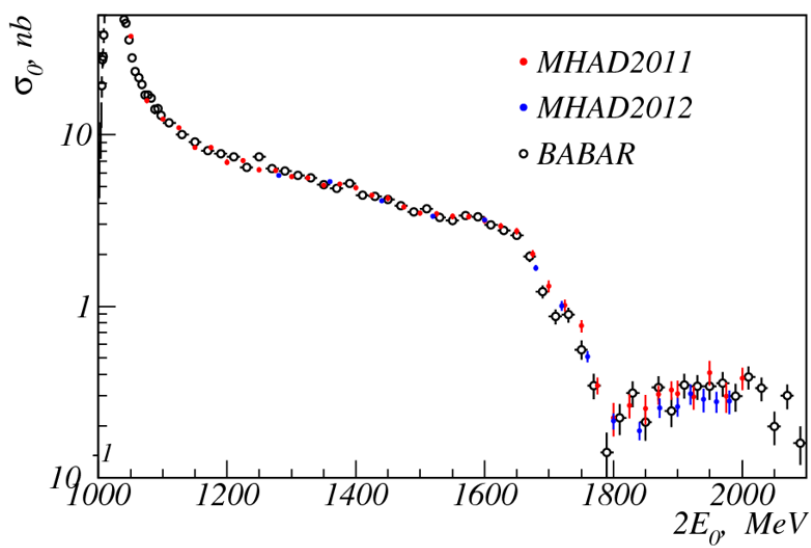

Figure 11. Preliminary results of measurement of the $e^{+} e^{-} \rightarrow$ $K^{+} K^{-}$cross section at SND.

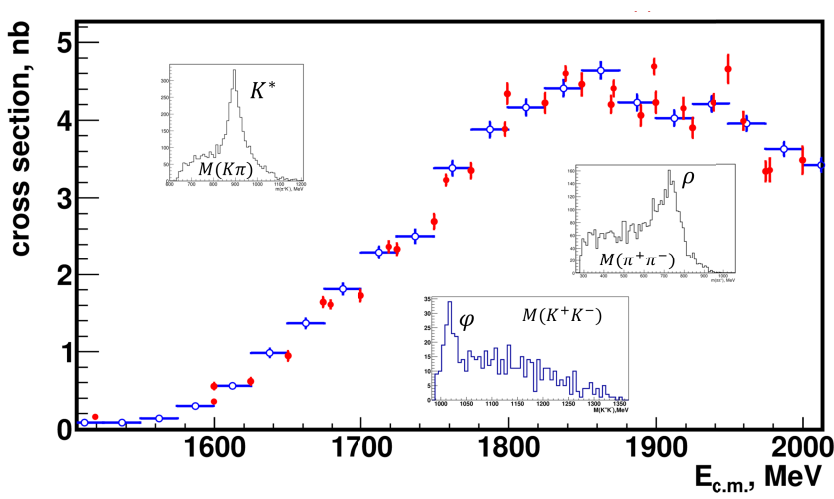

Figure 12. Preliminary results of measurement of the $e^{+} e^{-} \rightarrow$ $K^{+} K^{-} \pi^{+} \pi^{-}$cross section at CMD-3. Insets demonstrate some of the intermediate states observed in this process. 


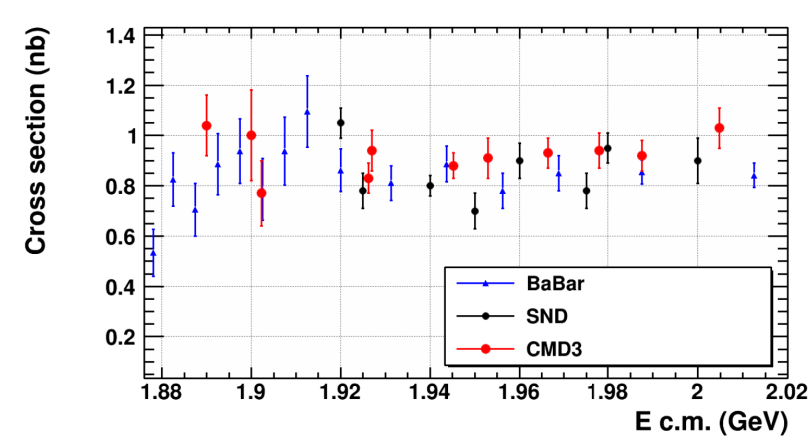

Figure 13. Preliminary results of measurement of the $e^{+} e^{-} \rightarrow p \bar{p}$ cross section at CMD-3 and SND, in comparison with BABAR data.

\subsection{Nucleon formfactor}

The maximum energy of VEPP-2000 is slightly above the $p \bar{p}$ and $n \bar{n}$ masses, making possible direct measurements of nucleon formfactors near threshold. Currently, about 6 $\mathrm{pb}^{-1}$ were collected by each detector at $\sqrt{s} \geq 2 m_{p}$.

The preliminary results of the $p \bar{p}$ production cross section measurements are shown in Fig. 13. Two separate techniques are used in the analysis. At energies $\sqrt{s}>1.92 \mathrm{GeV}$ proton and antiproton have enough kinetic energy to penetrate the wall of the vacuum chamber and be observed directly in the drift chamber with high efficiency. At smaller energies the analysis is more complicated, because both proton and antiproton stop in the wall and are not observed; thus the $p \bar{p}$ are identified by secondary particles characteristic of antiproton annihilation in the vacuum chamber wall.

The angular dependence of the $p \bar{p}$ production cross section allows to measure the $\left|G_{E} / G_{M}\right|$ ratio. The angular distributions of $G_{E}$ and $G_{M}$ terms differ at most at a zero angle, while both detectors detect $p$ and $\bar{p}$, mostly scattered at large angles. Therefore, while the collected luminosity is enough to reach a combined statistical error of $\sigma_{p \bar{p}}$ of about $2 \%$, the statistical error for the $\left|G_{E} / G_{M}\right|$ ratio is about $20 \%$ only. Both experiments at VEPP-2000 plan to collect significantly more statistics in this energy range, which will allow to measure $\left|G_{E} / G_{M}\right|$ with 5-10\% statistical precision.

The $e^{+} e^{-} \rightarrow n \bar{n}$ cross section was recently measured by SND [15] with about $25 \%$ statistical and $20 \%$ systematic errors (Fig. 14). The initial number of these events is about the same as the number of $e^{+} e^{-} \rightarrow p \bar{p}$, but $n \bar{n}$ events are much more difficult to identify. There is no signal from $n$, and $\bar{n}$ is identified by secondary particles, produced in annihilation, mostly detected in the calorimeter. It was observed, that the effective formfactors of proton and neutron have similar behavior near threshold.

\section{Conclusion}

After the VEPP-2000 collider was commissioned in Budker Institute of Nuclear Physics in 2010, two experiments, CMD-3 and SND, have collected more than $100 \mathrm{pb}^{-1}$ in

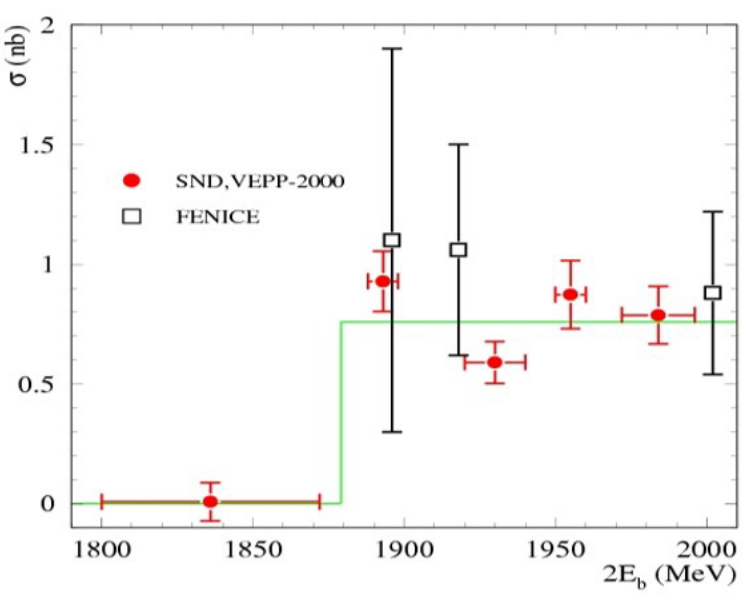

Figure 14. Cross section $e^{+} e^{-} \rightarrow n \bar{n}$, measured by SND.

the energy range $0.36 \leq \sqrt{s} \geq 2.0 \mathrm{GeV}$. Data analysis is in progress and the first results of measurements of cross sections $e^{+} e^{-} \rightarrow$ hadrons are already published by both groups. The collider operation was stopped in 2013 for an upgrade aimed at luminosity improvement up to 10 times at energies $\sqrt{s} \approx 2 \mathrm{GeV}$. Data taking is expected to resume in 2015 . The ultimate goal is to collect $1 \mathrm{fb}^{-} 1$ in two experiments.

\section{Acknowledgments}

This work is supported in part by the RFBR grants 12-02-00065-a, 12-02-01032-a, 12-02-01250-a. 12-0231498-mol-a, 12-02-31499-mol-a, 12-02-31501-mol-a, 13-02-00215-a, 13-02-00993, 14-02-91332 and DFG HA 1457/9-1.

\section{References}

[1] Yu. M. Shatunov et al., Conf. Proc. C 0006262, 439 (2000).

[2] D. Berkaev et al., Nucl. Phys. Proc. Suppl. 225-227, 303 (2012).

[3] B. I. Khazin et al., Nucl. Phys. Proc. Suppl. 181-182, 376 (2008).

[4] M. N. Achasov et al., Nucl. Instrum. Meth. A 598, 31 (2009).

[5] G. W. Bennett et al. (E821 Collaboration), Phys. Rev. D73, 072003 (2006).

[6] E. V. Abakumova et al., Nucl. Instrum. Meth. A 744, 35 (2014).

[7] K. Hagiwara, R. Liao, A. D. Martin, D. Nomura, T. Teubner, J. Phys. G 38, 085003 (2011).

[8] M. Davier, A. Hoecker, B. Malaescu, Z. Zhang1, Eur. Phys. J C 71, 1515 (2011), erratum Eur. Phys. J. C 72, 1874 (2012).

[9] T. Blum, A. Denig, I. Logashenko, E. de Rafael, B. Lee Roberts, T. Teubner and G. Venanzoni, arXiv:1311.2198 [hep-ph]. 
[10] M. N. Achasov et al. (SND Collaboration), Phys. Rev. D 66, 032001 (2002).

[11] B. Aubert et al. (BABAR Collaboration), Phys. Rev. D 70, 072004 (2004).

[12] R. R. Akhmetshin et al. (CMD-3 Collaboration), Phys. Lett. B 723, 82 (2013).
[13] A.E. Obrazovsky, S.I. Serednyakov, JETP Lett. 99, 363 (2014).

[14] M.N.Achasov et al. (SND Collaboration), Phys. Rev. D 63, 072002 (2001).

[15] M.N.Achasov et al. (SND Collaboration), Phys. Rev. D 90, 112007 (2014). 